\title{
VIII. Variably-coupled vibrations: II. Unequal masses or periods
}

\author{
Edwin H. Barton D.Sc. F.R.S. \& H. Mary Browning B.Sc.
}

To cite this article: Edwin H. Barton D.Sc. F.R.S. \& H. Mary Browning B.Sc. (1918) VIII. Variablycoupled vibrations: II. Unequal masses or periods, Philosophical Magazine Series 6, 35:205, 62-79, DOI: $10.1080 / 14786440108635737$

To link to this article: http://dx.doi.org/10.1080/14786440108635737

Published online: 08 Apr 2009.

Submit your article to this journal $\square$

Џlll Article views: 3

Q View related articles $\square$ 
maximum width of spark-gap used was $45 \mathrm{~mm}$., the spheres being $31 \mathrm{~mm}$. in diameter; so that according to tests made by C. Müller* the voltage applied could not have exceeded $50 \mathrm{KV}$. The K-series could not then have been excited, and it is probable that the wave-length excited belonged to the L-series of either metal.

I propose to investigate further the wave-lengths and other properties of these radiations. A report of such studies will be published shortly.

\section{Summary of Results.}

1. For the first time the excitation of a penetrating radiation by positive rays was observed. This effect was retained on photographic plates in the case of tin and lead, and it is surmised that it is the characteristic $\mathbf{X}$-radiation of these elements.

2. A lower limit was found to exist for the voltage necessary for excitation.

3. Einstein's quantum condition leads to the supposition that the new effect that has been observed is excitation

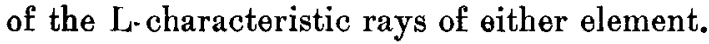

The Physical Laboratory,

Technical High School of Zurich.

August 1917.

VIII. Variably-Coupled Vibrations: II. Unequal Masses or Periods. By Edwin H. BaRTon, D.Sc., F.R.S., Professor of Physics, and H. MARY Browning, B.Sc., Lecturer and Demonstrator in Physics, University College, Nottinghamt.

[Plates I. \& II.]

$$
\text { Contents. }
$$

I. Introduction $\ldots \ldots \ldots \ldots \ldots \ldots \ldots \ldots \ldots \ldots, \ldots \ldots \ldots$

II. Theory for Unequat Masses ............ 63

Equations of Motion and Coupling.

Solution and Frequencies.

Initial Conditions.

Dampings, Separate and Coupled.

III. Theory for Unequal. Pariods $\ldots \ldots \ldots \ldots \ldots, 69$

Equations of Motion and Coupling.

Solution and Frequencies.

Initial Conditions.

IV. Relations among Variables $\ldots \ldots \ldots \ldots \ldots \ldots, \mathbf{7 2}$

* C. Müller, Ann. d. Phys. [4] xxviii. p. 585 (1910).

$\dagger$ Communicated by the Authors, 
V. Exphrimental Results .................. 76

Masses $20: 1$.

Logarithmic Decrements.

Masses $5: 1$.

Length $3: 4$.

VI. Summary

78

I. Introduction.

T $\mathrm{N}$ a recent paper * two types of coupled pendulums were experimented with, their lengths and the masses of their bobs being in each case equal. The present paper, the second of the series, deals with the double-cord pendulum only, but in cases where either the masses of the bobs are unequal or else the lengths of their suspensions are unequal.

These mechanical cases may he regarded as somewhat analogous to the electrical cases of inductively-coupled circuits with unequal inductances or unequal periods respectively.

With unequal masses and equal lengths it is noticeable that with small couplings a great increase in the amplitude of vibration of the small bob entailed very little loss in that of the large bob. Inde日d, for masses as 20:1 we almost realised the case of forced vibrations.

The funnel of the light bob was here of cardboard and so had an appreciable damping. This rendered it necessary to make corresponding modifications in the theory.

With unequal lengths and equal masses the response showed a great diminution for small couplings, whereas for larger couplings the mistuning seemed without appreciable effect.

The paper includes twenty-seven photographic reproductions of double sand traces obtained simultaneously one from each bob of the coupled pendulum.

\section{Theory for Unequal Masses.}

Equations of Motion and Coupling.--Throughout the work described in the present paper the double-cord pendulum was used. This was shown in figs. 1, 2, and 4 of the first paper. The equations of motion and coupling were given as (27)-(29) and may now be rewritten here as follows :-

$$
\left.\begin{array}{l}
\mathrm{P} \frac{d^{2} y}{d t^{2}}+\frac{\mathrm{P}+\mathrm{Q}+\beta \mathrm{Q}}{(1+\beta)(\mathrm{P}+\mathrm{Q})} \mathrm{P} \frac{g}{l} y=\frac{\beta}{(1+\beta)} \cdot \frac{\mathrm{PQ}}{(\mathrm{P}+\mathrm{Q})} \frac{g}{l} z \\
\mathrm{Q} \frac{d z^{2}}{d t^{2}}+\frac{\mathrm{P}+\beta \mathrm{P}+\mathrm{Q}}{(1+\beta)(\mathrm{P}+\mathrm{Q})} \mathrm{Q} \frac{g}{l} z=\frac{\beta}{(1+\beta)} \cdot \frac{\mathrm{PQ}}{(\mathrm{P}+\mathrm{Q})} \frac{g}{l} y
\end{array}\right\} .
$$

and

$$
\gamma^{2}=\frac{\beta^{2} \mathrm{PQ}}{(\mathrm{P}+\mathrm{Q}+\beta \mathrm{Q})(\mathrm{P}+\beta \mathrm{P}+\mathrm{Q})} \cdot \text {. . . }
$$

* Phil. Mag. (6) vol. xxxiv. no. 202, pp. 246-270 (Oct. 1917). 
Let us now write in the above

$$
\frac{\mathrm{Q}}{\mathrm{P}}=\rho, \quad \frac{q}{l}=m^{2} . \quad . \quad \cdot \quad \cdot \quad .
$$

Also divide the two equations (1) by $\mathrm{P}$ and $\mathrm{Q}$ respectively, and insert the frictional term $2 k d y \mid d i$ in the first of them. We then obtain

$$
\begin{aligned}
\frac{d^{2} y}{d t^{2}}+2 k \frac{d y}{d t}+\frac{1+\rho+\beta \rho}{(1+\beta)(1+\rho)} m^{2} y & =\frac{\beta}{(1+\beta)} \cdot \frac{\rho}{(1+\rho)} m^{2} z \\
\frac{d^{2} z}{d t^{2}}+\frac{1+\rho+\beta}{(1+\beta)(1+\rho)} m^{2} z & =\frac{\beta}{(1+\beta)} \cdot \frac{1}{(1+\rho)} m^{2} y .
\end{aligned}
$$

These may be written

and

$$
\frac{d^{2} y}{d t^{2}}+2 k \frac{d y}{d t}+a y=\rho b z, \quad \text {. . . . }
$$

$$
\frac{d^{2} z}{d t^{2}}+c z=b y, \quad \text {. . . . . . . }
$$

where

and

$$
\left.\begin{array}{c}
a=\frac{1+\rho+\beta \rho}{(1+\beta)(1+\rho)} m^{2}, b=\frac{\beta m^{2}}{(1+\beta)(1+\rho)}, \\
c=\frac{1+\rho+\beta}{(1+\beta)(1+\rho)} m^{2}
\end{array}\right\} .
$$

Solution and Frequencies.--To solve (6) and (7) let us write

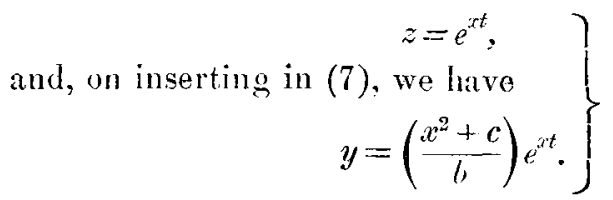

Then (9) substituted in (6) gives

or

$$
\begin{gathered}
\left(\frac{x^{2}+c}{b}\right)\left(x^{2}+2 k x+a\right)=\rho b \\
x^{4}+2 k x^{3}+(c+a) x^{2}+2 k c x+c a-\rho b^{2}=0,
\end{gathered}
$$

which is the auxiliary biquadratic in $x$. Though this equation has the form of the general biquadratic, an approximate solution, presenting all the accuracy needed for our purpose, may be easily obtained by noting that $k$ is small compared with the other constants. For, as appears from the experiments, $k$ is of the order one-thousandth of the coefficient of $x^{2}$ and of the constant term.

Then we may write for the roots of $x$ in the biquadratic (10) the values

$$
-r \pm i p \text { and }-s \pm i q, . . .
$$


where $i$ denotes $\mathcal{V}(-1)$, and $r$ and $s$ (being comparable to $k$ ) are to be treated as small quantities whose squares or products are negligible in comparison with $p$ and $q$ which depend upon the larger constants of the equation.

Thus, with the roots from (11) we may write instead of (10) the equivalent equation

$$
\begin{aligned}
& (x+r-i p)(x+r+i p)(x+s-i q)(x+s+i q)=0, \\
& \text { or } x^{4}+2(r+s) x^{3}+\left(p^{2}+q^{2}+r^{2}+s^{2}+4 r s\right) x^{2} \\
& +2\left(p^{2} s+q^{2} r+r^{2} s+r s^{2}\right) x+\left(p^{2}+r^{2}\right)\left(q^{2}+s^{2}\right)=0 .
\end{aligned}
$$

This, on omitting the negligible quantities, becomes the approximate equation sufficiently accurate for our purpose,

$$
x^{4}+2(r+s) x^{3}+\left(p^{2}+q^{2}\right) x^{2}+2\left(p^{2} s+q^{2} r\right) x+p^{2} q^{2}=0 \text {. (13) }
$$

The comparison of coefficients in (10) and (13) yields

$$
\begin{aligned}
& r+s=k, \\
& p^{2}+q^{2}=c+a, \quad . \quad . \quad . \quad . \quad . \\
& p^{2} s+q^{2} r=c k, \text {. . . . . . . } \\
& p^{2} q^{2}=c a-\rho b^{2} .
\end{aligned}
$$

From (15) and (17) we may eliminate $q^{2}$ and obtain a quadratic in $p^{2}$ whose roots may be called $p^{2}$ and $q^{2}$. We thus find

and

$$
\left.\begin{array}{l}
2 p^{2}=c+a+\sqrt{ }\left\{(a-c)^{2}+4 \rho b^{2}\right\} \\
2 q^{2}=c+a-\sqrt{ }\left\{(a-c)^{2}+4 \rho b^{2}\right\}
\end{array}\right\} .
$$

Again, from (14) and (16) we obtain

$$
r=\frac{p^{2}-c}{p^{2}-q^{2}} k \text { and } s=\frac{c-q^{2}}{p^{2}-q^{2}} k
$$

And by use of (18) these become

and

$$
\begin{aligned}
& r=\frac{a-c+\sqrt{ }\left\{(a-c)^{2}+4 \rho b^{2}\right\}}{2 \sqrt{ }\left\{(a-c)^{2}+4 \rho b^{2}\right\}} k . . . \\
& s=\frac{c-a+\sqrt{ }\left\{(a-c)^{2}+4 \rho b^{2}\right\}}{2 \sqrt{ }\left\{(a-c)^{2}+4 \rho b^{2}\right\}} k . . .
\end{aligned}
$$

Then, inserting the values of $a, b$, and $c$ from (8) in (18), (19), and (20), we obtain

$$
\begin{aligned}
& p=m, \quad q=\frac{m}{\sqrt{ }(1+\beta)}, \quad . \quad . \quad . \quad \text {. } \\
& \frac{p}{q}=\sqrt{ }(1+\beta), \quad \text {. . . . . } \\
& r=\frac{\rho k}{1+\rho}, \text {. . . . . . . } \\
& s=\frac{k}{1+\rho} \cdot \text {. . . . . . . . }
\end{aligned}
$$

Phil. Mag. S. 6. Vol. 35. No. 205. Jan. $1918 . \quad$ F 
Thus, using (11) in (9) and introducing the usual constants, the general solution may be written in the form

$$
z=e^{-r t}\left(\mathrm{~A} e^{p i t}+\mathrm{B} e^{-p i t}\right)+e^{-s t}\left(\mathrm{C} e^{q i t}+\mathrm{D} e^{-q i t}\right), .
$$

and, omitting $r^{2}$ and $s^{2}$,

$$
\begin{aligned}
y & =\frac{\left(-p^{2}+c\right)}{b} e^{-r t}\left(\mathrm{~A} e^{p i t}+\mathrm{B} e^{-p i t}\right)+\frac{\left(-q^{2}+c\right)}{b} e^{-s t}\left(\mathrm{C} e^{q i t}+\mathrm{D} e^{-q i t}\right) \\
& +\frac{2 p r i}{b} e^{-r t}\left(-\mathrm{A} e^{p i t}+\mathrm{B} e^{-p i t}\right)+\frac{2 q s i}{b} e^{-s t}\left(-\mathrm{C} e^{q i t}+\mathrm{D} e^{-q i t}\right)
\end{aligned}
$$

Or, by transformation of (25) and (26) and use of (21)(24), we may write the general solution in the form

and

$$
z=\mathrm{E} e^{-\rho s t} \sin (m t+\epsilon)+\mathrm{F} e^{-s t} \sin \left(\frac{m t}{\sqrt{ }(1+\beta)}+\phi\right),
$$

$y=-\rho \mathrm{E}^{\prime} e^{-\rho s t} \sin \left(m t+\epsilon^{\prime}\right)+\mathrm{F}^{\prime} e^{-s t} \sin \left(\frac{m t}{\sqrt{ }(1+\beta)}+\phi^{\prime}\right)$,

where

and

$$
\left.\begin{array}{c}
\left(\mathrm{E}^{\prime}\right)^{2}=\mathrm{E}^{2} \frac{\beta^{2} m^{2}+4(1+\beta)^{2} k^{2}}{\beta^{2} m^{2}} \\
\tan \left(\epsilon^{\prime}-\epsilon\right)=\frac{2(1+\beta) k}{\beta m} ;
\end{array}\right\}
$$

also

and

$$
\left.\left(\mathrm{F}^{\prime}\right)^{2}=\mathrm{F}^{2} \frac{\beta^{2} m^{2}+4(1+\beta) k^{2}}{\beta^{2} m^{2}}\right)
$$

$$
\left.\tan \left(\phi^{\prime}-\phi\right)=\frac{-2 \sqrt{ }(1+\beta) k}{\beta m} ;\right\}
$$

the exponential coefficient $s$ being given by (24), and $F, \epsilon$, $F$, and $\phi$ being the arbitrary constants dependent on the initial conditions. In many of the experimental cases $\mathbf{E}^{\prime}$ may be assimilated to $E$ and $F^{\prime}$ to $F$ without appreciable error. The changes $\left(\epsilon^{\prime}-\varepsilon\right)$ and $\left(\phi^{\prime}-\phi\right)$ of the phase angles may be distinctly appreciable for very small values of $\beta$. But in these cases the vibrations show a slow waxing and waning of amplitude and the phase is of very little importance. On the other hand, for $\beta$ equal to unity, we have

$$
\tan \left(\epsilon^{\prime}-\epsilon\right)=4 \pi / m \text { and } \tan \left(\phi^{\prime}-\phi\right)=-2 \sqrt{ } 2 k / m \text {. }
$$

And the numerical values of these are of the order 0.020 and 0.014 , hence $\epsilon^{\prime}-\epsilon=1^{\circ} 10^{\prime}$ and $\phi^{\prime}-\phi=0^{\circ} 48^{\prime}$ nearly. Hence for all our present experimental cases, we may drop the four accents in equation (28). 
Coupled Vibrations: Unequal Masses or Periods.

Initial Conditions. Case I.--Suppose the heavy bob of mass $\mathrm{Q}$ (which $=\rho \mathrm{P}$ ) is pulled aside and that the light one of mass $\mathrm{P}$ is allowed to bang at rest in its more or less displaced position according to the coupling in use. Then wo may write :

For $t=0$ let $z=f$,

then it follows statically that $y=\frac{\beta \rho f}{1+\rho+\beta p}$; also put

$$
\frac{d z}{d t}=0 \quad \text { and } \quad \frac{d y}{d t}=0
$$

Differentiating with respect to time (27) and (28) without its accents, and writing in the latter $n$ for $m / \sqrt{ }(1+\beta)$, we find

$$
\begin{aligned}
& \frac{d z}{d t}=\mathrm{E} e^{-\rho s t}[m \cos (m t+\epsilon)-\rho s \sin (m t+\epsilon)] \\
&+\mathrm{F} e^{-s t}[n \cos (n t+\phi)-s \sin (n t+\phi)], \\
& \frac{d y}{d t}=-\rho \mathrm{E} e^{-\rho s t}[m \cos (m t+\epsilon)-\rho s \sin (m t+\epsilon)] \\
&+\mathrm{F} e^{-s t}[n \cos (n t+\phi)-s \sin (n t+\phi)] .
\end{aligned}
$$

The conditions (31) introduced in equations (27), (28), (32), and (33) give

$$
\begin{gathered}
f=\mathrm{E} \sin \epsilon+\mathrm{F} \sin \phi, \\
\frac{\beta \rho f}{1+\rho+\beta \rho}=-\rho \mathrm{E} \sin \epsilon+\mathrm{F} \sin \phi, . \\
0=\mathrm{E}(m \cos \epsilon-\rho s \sin \epsilon)+\mathrm{F}(n \cos \phi-s \sin \phi), . \\
0=-\rho \mathrm{E}(m \cos \epsilon-\rho s \sin \epsilon)+\mathrm{F}(n \cos \phi-s \sin \phi) .
\end{gathered}
$$

But, by reason of the smallness of $\rho s$ in comparison with $m$ (of the order 0.01 ) and of $s$ in comparison with $n$ (still less), we may write instead of (36) and (37) the following:

$$
\text { and } \quad \begin{aligned}
& 0=\mathrm{E} m \cos \epsilon+\mathrm{F} n \cos \phi, \quad . \quad . \quad . \\
& 0=-\rho \mathrm{E} m \cos \epsilon+\mathrm{F} n \cos \phi . \quad . \quad .
\end{aligned}
$$

These are satisfied by

$$
\epsilon=\frac{\pi}{2} \quad \text { and } \quad \phi=\frac{\pi}{2} \ldots . . . .
$$

These values inserted in (34) and (35) give

and

$$
f=\mathrm{E}+\mathrm{F} \text {, }
$$

whence

$$
\frac{\beta \rho f}{1+\rho+\beta \rho}=-\rho \mathrm{E}+\mathrm{F} \text {; }
$$

$$
\mathrm{E}=\frac{f}{1+\rho+\beta \rho} \quad \text { and } \quad \mathrm{F}=\frac{(1+\rho) \beta f}{1+\rho+\beta \rho}
$$


Hence, for the special solution with these initial conditions, we have

$z=\frac{f}{1+\rho+\beta \rho} e^{-\rho s t} \cos m t+\frac{(1+\rho) \beta f}{1+\rho+\beta \rho} e^{-s t} \cos \frac{m t}{\sqrt{ }(1+\beta)}$,
$y=-\frac{\rho f}{1+\rho+\beta \rho} e^{-\rho s t} \cos m t+\frac{(1+\rho) \beta f}{1+\rho+\beta \rho} e^{-s t} \cos \frac{m t}{\sqrt{ }(1+\beta)}$,

where $s=\frac{k}{1+\rho}$.

Thus the ratios of the amplitudes of the quick and slow components in the $y$ and $z$ vibrations are respectively given by

$$
\frac{-\rho}{(1+\rho) \beta} e^{-(\rho-1) s t} \text { and } \frac{1}{(1+\rho) \beta} e^{-(\rho-1) s t} \text {. }
$$

Case II.-Suppose now that the heavy bob (of mass $\mathrm{Q}=\rho \mathrm{P}$ ) is pulled aside while the light one (of mass $\mathrm{P}$ ) is held undisplaced. Then we have:

For

$$
\left.\begin{array}{c}
t=0, \quad z=f, \quad y=0, \\
\frac{d y}{d t}=0 \text { and } \frac{d z}{d t}=0 .
\end{array}\right\} .
$$

Putting (44) in (27), (28) without accents, (32) and (33), and omitting small quantities as before, we find

$$
\begin{aligned}
& \left.\begin{array}{l}
f=\mathrm{E} \sin \epsilon+\mathrm{F} \sin \phi, \\
0=-\rho \mathrm{E} \sin \epsilon+\mathrm{F} \sin \phi ;
\end{array}\right\} \\
& 0=\mathrm{E} m \cos \epsilon+\mathrm{F} n \cos \phi \text {, } \\
& 0=-\rho \mathrm{E} m \cos \epsilon+\mathrm{F} n \cos \phi .\}
\end{aligned}
$$

Then (46) is satisfied by

$$
\epsilon=\frac{\pi}{2} \quad \text { and } \quad \phi=\frac{\pi}{2} \text {, }
$$

and putting these values in (45), we obtain

$$
\mathrm{E}=\frac{f}{1+\rho} \text { and } \mathrm{F}=\frac{\rho f}{1+\rho} .
$$

Hence, for the special solution with these initial conditions, wo have

$$
\begin{aligned}
& y=-\frac{\rho f}{1+\rho} e^{-\rho s t} \cos m t+\frac{\rho f}{1+\rho} e^{-s t} \cos \frac{m t}{\sqrt{ }(1+\beta)}, \\
& z=\frac{f}{1+\rho} e^{-\rho s t} \cos m t+\frac{\rho f}{1+\rho} e^{-s t} \cos \frac{m t}{\sqrt{ }(1+\beta)} .
\end{aligned}
$$


Coupled Vibrations: Unequal Masses or Periods.

Accordingly the ratios of the amplitudes of the quick and slow vibrations in the $y$ and $z$ traces are respectively

$$
-e^{-(\rho-1) s t} \text { and } \frac{e^{-(\rho-1) s t}}{\rho} \cdot . . .
$$

Relation of Dampings in the Vibrations separate and coupled.-The vibrations of a separate damped pendulum of length $l$ are derived from the equation of motion

where $m^{2}=g / l$.

$$
\frac{d^{2} y}{d t^{2}}+2 k \frac{d y}{d t}+m^{2} y=0, \quad \text {. . . . . }
$$

The solution of this involves simple harmonic vibrations of approximate period

and of damping factor

$$
\tau=2 \pi / m,
$$

$$
e^{-k t} \text {. }
$$

Thus the ratio of successive amplitudes is

noarly.

$$
e^{k \pi / 2}=e^{k \pi / m}
$$

But the logarithmic decrement $\lambda$ (per half wave) is defined as the logarithm to base $e$ of this ratio.

Hence

$$
\lambda=\frac{k \pi}{m} \text { or } k=\frac{m \lambda}{\pi}, \ldots . . .
$$

which gives the relation between damping coefficient and logarithmic decrement for a separato pendulum.

We have now to express in terms of $\lambda$ the two damping coefficients $r$ and $s$ which apply to the superposed vibrations when the pendulums are coupled. Thus, combining (23) and (24) with (51), we find

and

$$
r=\frac{\rho}{1+\rho} \cdot \frac{m \lambda}{\pi}, . . . . . . . .
$$

$$
s=\frac{1}{1+\rho} \cdot \frac{m \lambda}{\pi} . \quad . \quad . \quad . \quad . \quad .
$$

III. 'l'heory for Unequal Periods.

Equations of Motion and Coupling.-Still using the double-cord pendulum, as shown in figs. 1, 2, and 4 of the first paper, we now make the masses of the bobs equal, but the lengths of the suspensions unequal. (The droops of the two bridles always remain equal.) In other words, $Q=P$ or $\rho=1$, while the lengths of the suspensions for the 
$y$ and $z$ vibrations are now denoted by $\eta l$ and $l$ respectively, the droop of each bridle being $\beta l$ as before.

Then the equations of motion of the pendulums may be written at first in the form :

$$
\begin{aligned}
& \mathrm{P} \frac{d^{2} y}{d t^{2}}+\mathrm{P} g \theta=0, \ldots . \\
& \mathrm{Q} \frac{d^{2} z}{d t^{2}}+\mathrm{Q} g \Psi=0, . . \\
& \hline
\end{aligned}
$$

where $\theta$ and $\psi$ are the inclinations of the suspensions to the vertical.

But we have also

$$
\theta=\frac{y-\beta l \omega}{\eta l} \text { and } \psi=\frac{z-\beta l \omega}{l}, \quad . \quad .
$$

where $\omega$ is the inclination to the vertical of the planes of the bridles.

Neglecting masses of bridles, connector, and suspensions, $\omega$ must satisfy

$$
\mathrm{Q} g(\psi-\omega)=\mathrm{P} g(\omega-\theta)=\mathrm{P} g(\psi-\omega) . \quad .
$$

Then (56) in (57) gives

$$
l_{\omega}=\frac{y+\eta z}{\beta+\beta \eta+2 \eta}, . . . . .
$$

And (58) in (56) yields

$$
\theta=\frac{(2+\beta) y-\beta z}{l(\beta+\beta \eta+2 \eta)} \text { and } \psi=\frac{(\beta+2 \eta) z-\beta y}{l(\beta+\beta \eta+2 \eta)} .
$$

Then by (59), equations (54) and (55) become

$$
\begin{aligned}
& \frac{d^{2} y}{d t^{2}}+\frac{2+\beta}{\beta+\beta \eta+2 \eta} m^{2} y=\frac{\beta n^{2}}{\beta+\beta \eta+2 \eta} z, . \\
& \frac{d^{2} z}{d t^{2}}+\frac{\beta+2 \eta}{\beta+\beta \eta+2 \eta} m^{2} z=\frac{\beta m^{2}}{\beta+\beta \eta+2 \eta} y, . .
\end{aligned}
$$

where $m^{2}$ is written for $g / l$.

So, for the coupling $\gamma$, we have

$$
\gamma^{2}=\frac{\beta^{2}}{(2+\beta)(\beta+2 \eta)} \cdot \text {. . . . . }
$$

Hence, for $\eta=1$, we recover the original relation

$$
\gamma=\frac{\beta}{2+\beta}, \text {. . . . . . . . }
$$

which agrees with (32) of the first paper. 
Coupled Vibrations: Unequal Masses or Periods.

Solution and Frequencies.-In equation (61) try

then we have

$$
\left.y=\frac{x^{2}(\beta+\beta \eta+2 \eta)+(\beta+2 \eta) m^{2}}{\beta m^{2}} e^{x t}\right\} .
$$

And, by (64) in (60), we obtain

$$
\begin{gathered}
\left\{x^{2}(\beta+\beta \eta+2 \eta)+(\beta+2 \eta) m^{2}\right\}\left\{x^{2}(\beta+\beta \eta+2 \eta)\right. \\
\left.+(2+\beta) m^{2}\right\}=\beta^{2} m^{4} .
\end{gathered}
$$

This reduces to the auxiliary biquadratic in $x$,

$$
x^{4}(\beta+\beta \eta+2 \eta)+2(1+\beta+\eta) m^{2} x^{2}+2 m^{4}=0 . \quad .
$$

Solving this as a quadratic in $x^{2}$, we have

$$
x^{2}=-m^{2} \frac{1+\beta+\eta \pm \sqrt{ }\left\{(1-\eta)^{2}+\beta^{2}\right\}}{\beta+\beta \eta+2 \eta} . \text {. }
$$

Or, let us write

$$
x= \pm p i \text { or } \pm q i
$$

Then, for the sake of brevity putting $\Delta^{2}$ for $(1-\eta)^{2}+\beta^{2}$, we have

and

$$
\left.\begin{array}{l}
p^{2}=\frac{1+\beta+\eta+\Delta}{\beta+\beta \eta+2 \eta} m^{2}, \\
q^{2}=\frac{1+\beta+\eta-\Delta}{\beta+\beta \eta+2 \eta} m^{3}, \\
\frac{p}{q}=\left\{\frac{1+\beta+\eta+\Delta}{1+\beta+\eta-\Delta}\right\}^{1 / 2}
\end{array}\right\}
$$

Thus, using (67) in (64) and introducing the usual constants, we obtain

and

$$
z=\mathrm{E} \sin (p t+\epsilon)+\mathrm{F} \sin (q t+\phi), \quad . \quad .
$$

$y=-\frac{1-\eta+\Delta}{\beta} \mathrm{E} \sin (p t+\epsilon)+\frac{\Delta-(1-\eta)}{\beta} \mathrm{F} \sin (q t+\phi),(70)$ $p$ and $q$ being defined by (68).

Initial Conditions. - Consider the case of pulling aside the bob $Q$ of the pendulum of length $l$ whose vibrations are denoted by $z$, the other bob hanging at rest in a more or less displaced position according to the magnitude of the coupling. 
Thus, we may write :

For $\quad t=0, \quad z=f$,

when it follows statically that, $y=\frac{\beta f}{2+\beta}$,

and we have also $\quad \frac{d y}{d t}=0, \quad \frac{d z}{d t}=0$.

Differentiating (69) and (70) with respect to the time, and introducing (71) gives equations which are satisfied by

$$
\epsilon=\frac{\pi}{2} \text { and } \phi=\frac{\pi}{2} \text {. . . . . . . }
$$

Then, introducing (71) and (72) in (69) and (70) we find

$$
\left.\begin{array}{l}
\mathrm{E}=\frac{(2+\beta)(-1+\eta+\Delta)-\beta^{2}}{2(2+\beta) \Delta} f, \\
\mathrm{~F}=\frac{(2+\beta)(1-\eta+\Delta)+\beta^{2}}{2(2+\beta) \Delta} f_{.}
\end{array}\right\} . \cdot
$$

Finally, (72) and (73) in (69) and (70) give as the required special solution

and

$$
\begin{aligned}
z & =\frac{(2+\beta)(-1+\eta+\Delta)-\beta^{2}}{2(2+\beta) \Delta} f \cos p t \\
& +\frac{(2+\beta)(1-\eta+\Delta)+\beta^{2}}{2(2+\beta) \Delta} f \cos q t,
\end{aligned}
$$

$$
\begin{aligned}
y= & -\frac{1+\beta+\eta-\Delta}{2(2+\beta) \Delta} \beta f \cos p t \\
& +\frac{1+\beta+\eta+\Delta}{2(2+\beta) \Delta} \beta f \cos q t . . . .
\end{aligned}
$$

IV. Relations among Vartables.

It is instructive to plot graphs with the values of the coupling $\gamma$ as ordinates, the absciss $\infty$ being the corresponding ralues of $\beta$ (ratio of droop of bridle to pendulum length). A different graph is needed for each value of $\eta$ and $\rho$ (which are respectively the ratios of pendulum lengths and masses of bobs).

The data for these graphs are derived from the equations and are given in Tables I., II., and III. (and Table I. p. 265 of October paper). 
Table I.-Masses 20:1 and lengths equal

\begin{tabular}{|c|c|c|c|}
\hline $\begin{array}{c}\text { Coupling } \\
\gamma .\end{array}$ & $\begin{array}{c}\text { Bridle Droop } \\
\text { Pendulum length. }\end{array}$ & $\begin{array}{l}\text { Actual Droop } \\
\text { for total length } \\
229 \mathrm{~cm} .\end{array}$ & $\begin{array}{c}\text { Frequency } \\
\text { Ratio } \\
p: q=\sqrt{ }(1+\beta) .\end{array}$ \\
\hline $\begin{array}{c}\text { Per cent. } \\
0 \\
1 \\
2 \\
3 \\
4 \\
5 \\
10 \\
20 \\
30 \cdot 4\end{array}$ & $\begin{array}{l}0 \cdot 0 \\
0 \cdot 05 \\
0 \cdot 10 \\
0 \cdot 151 \\
0.207 \\
0.265 \\
0.60 \\
1.53 \\
3.00\end{array}$ & $\begin{array}{r}\text { cm. } \\
0.0 \\
10.9 \\
20.8 \\
29 \cdot 9 \\
39 \cdot 7 \\
48 \cdot 1 \\
85.9 \\
138.6 \\
171 \cdot 8\end{array}$ & $\begin{array}{l}1 \cdot 00 \\
1.025 \\
1 \cdot 05 \\
1.07 \\
1 \cdot 10 \\
1 \cdot 12 \\
1 \cdot 27 \\
1 \cdot 59 \\
200\end{array}$ \\
\hline
\end{tabular}

Table II.-Masses 5 : 1 and lengths equal.

\begin{tabular}{|c|c|c|c|}
\hline $\begin{array}{c}\text { Coupling } \\
\gamma .\end{array}$ & $\underset{\text { Pendulum length. }}{\text { Bridle Droop }} \beta$ & $\begin{array}{l}\text { Actual Droop } \\
\text { for total length } \\
229 \mathrm{~cm} .\end{array}$ & $\begin{array}{c}\text { Frequency } \\
\text { Ratio } \\
p: q=\sqrt{ }(1+\beta) .\end{array}$ \\
\hline $\begin{array}{c}\text { Per cent. } \\
0 \\
1 \\
2 \\
5 \cdot 2 \\
9 \cdot 4 \\
25 \cdot 5 \\
39 \cdot 5 \\
48 \cdot 8\end{array}$ & $\begin{array}{l}0 \\
0 \cdot 027 \\
0 \cdot 058 \\
0 \cdot 141 \\
0 \cdot 259 \\
1 \\
2 \\
3\end{array}$ & $\begin{array}{r}\mathrm{cm} . \\
0 \\
6 \cdot 0 \\
13 \cdot 3 \\
28 \cdot 2 \\
47 \cdot 1 \\
114.5 \\
152 \cdot 7 \\
171 \cdot 8\end{array}$ & $\begin{array}{l}1 \cdot 00 \\
1 \cdot 014 \\
1 \cdot 029 \\
1 \cdot 07 \\
1 \cdot 12 \\
1 \cdot 414 \\
1 \cdot 732 \\
2\end{array}$ \\
\hline
\end{tabular}

TABLE III.-Masses equal aud lengths $3: 4$.

\begin{tabular}{|c|c|c|c|}
\hline $\begin{array}{c}\text { Coupling } \\
\gamma .\end{array}$ & $\begin{array}{c}\text { Bridle Droop } \\
\text { Pendulum length. }\end{array}$ & $\begin{array}{c}\text { Actual Droop } \\
\text { for total length } \\
229 \mathrm{~cm} .\end{array}$ & $\begin{array}{c}\text { Frequency } \\
\text { Ratio } \\
p: q .\end{array}$ \\
\hline \begin{tabular}{c|c} 
Per cent. \\
0
\end{tabular} & 0 & $\mathbf{c m .}$ & \\
5.5 & $0 \cdot 1$ & 0 & $1 \cdot 154$ \\
$10 \cdot 3$ & $0 \cdot 2$ & $20 \cdot 8$ & $1 \cdot 16$ \\
$22 \cdot 4$ & 0.5 & $38 \cdot 2$ & $1 \cdot 175$ \\
36.5 & $\mathbf{1}$ & $76 \cdot 3$ & $1 \cdot 29$ \\
$59 \cdot 9$ & $2 \cdot 6$ & $114 \cdot 5$ & $1 \cdot 48$ \\
$63 \cdot 2$ & 3 & 165.4 & $2 \cdot 00$ \\
& & $171 \cdot 8$ & $2 \cdot 11$ \\
\hline
\end{tabular}


The graphs referred to are given in fig. 1 .

We may now, from the data in the same tables, plot graphs with the values of the frequency ratios $p: q$ as ordinates, the abscissæ being the corresponding values of the coupling $\gamma$.

These are shown in fig. 2, separate graphs being plotted for mass ratios 1,5 , and 20 and lengths equal, and also for lengths $3: 4$ and masses equal.

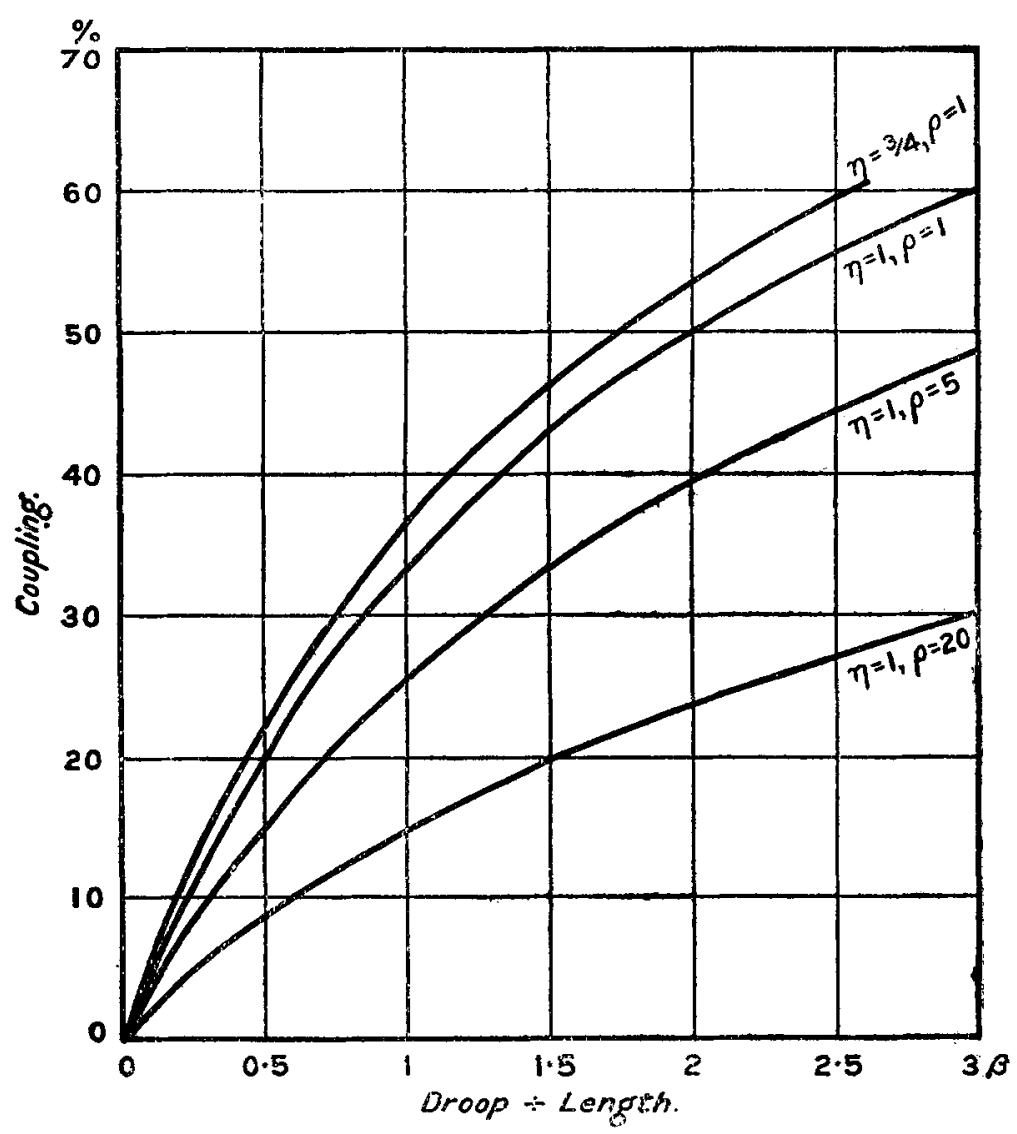

Fig. 1.-Couplings and Droop.

With the separate frequencies equal and a given coupling, it may be noted that the greater the inequality of the masses the greater is the inequality of the frequencies of the resulting superposed vibrations of the coupled system. 
Coupled Vibratıons : Unequal Masses or Periods. 75

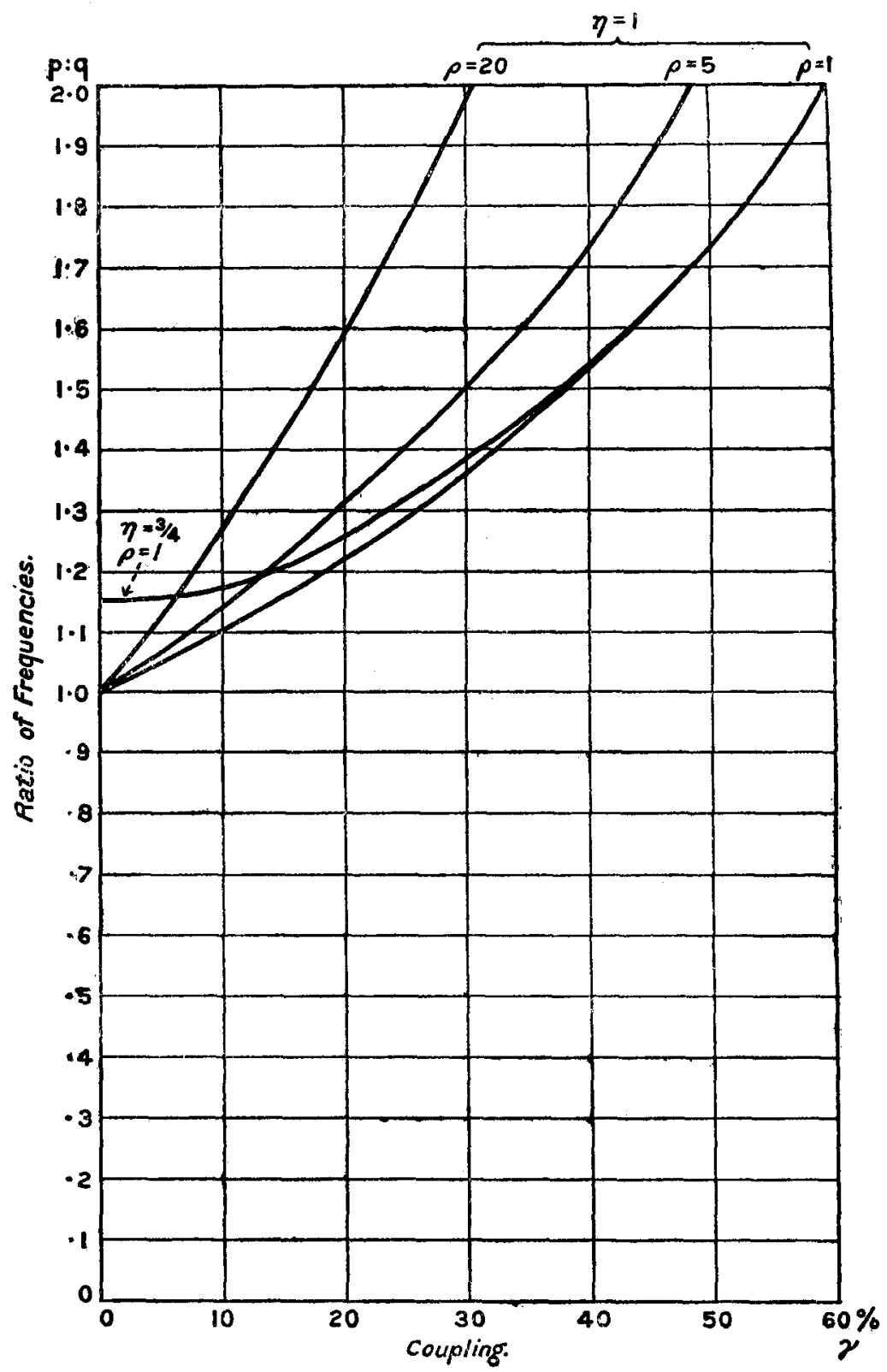

Fig. 2.-Frequency Ratios and Coupling. 
When the coupling vanishes the frequencies of the separate vibrations are of course undisturbed. Thus for equal lengths, but any ratio of masses, we have for $\gamma=0, p: q$ equals unity. But for different separate frequencies (i. e., $\eta$ not equal to unity) we have for $\gamma=0, p: q$ greater than unity. But with large couplings the effect of unequal separate frequencies gradually disappears.

\section{Experimental Results.}

Masses $20: 1$.- The bobs used in these experiments were of the order $1000 \mathrm{gms}$. and $50 \mathrm{gms}$. respectively. Figs. 1-11 of Plate I. give photographic reproductions of the double sand traces simultaneously obtained when the masses of the bobs $\mathrm{Q}$ and $\mathrm{P}$ were as $20: 1$, i. e., $\rho=20$.

The couplings vary from 1 per cent. in the first to a little over 30 per cent. in the last, and are shown as percentages on every figure.

Figs. 1-8 were obtained by drawing the heavy bob aside horizontally, the light bob being allowed to bang at rest in its more or less displaced position according as the coupling was tight or loose. In figs. 9-11, while the heavy bob was pulled aside, the light one was held in its undisplaced position. Figs. 1-6 show a very marked effect due to the inequality of the masses. For, as the resultant vibrations of the light bob wax and wane in amplitude, those of the heavy bob scarcely change. Thus showing that with masses $20: 1$ we have in this respect almost reached the limiting case of forced vibrations in which the reaction of the driven on the driver is negligible. The frequencies, however, are still appreciably affected. 'The contrast with the case of equal masses may be seen by referring to figs. 1-5 in Plate V. of the October paper, where the waxings and wanings occur equally and alternately in both traces. Figs. 1-8 show that as the coupling increases the inequality of the frequencies of the superposed vibrations increases also. Hence there are fewer vibrations in the beat cycle and this fulfils the theory.

In fig. 9 the initial displacement of the heavy bob was so great that a collision occurred between the two as indicated. But its effect passed away after a few vibrations. This may be seen by fig. 10 , in which with a slightly smaller displacement the collision was avoided.

Fig. 11 shows appreciable damping of the vibration of the light bob which was held undisplaced while the heavy one was drawn aside, whereas that of the heavy bob is not appreciably damped. This is exactly what might be expected 
from general considerations. But it seems at first sight in direct contradiction to the theory which shows that the $y$ and $z$ vibrations for the light and heavy bobs respectively involve the selfsame damping factors. But by equations (23) and (24) we see that one damping coefficient is $\rho$-times the other. Again, by equation (48) the amplitude of the slow vibrations of the heavy bob is $\rho$-times that of its quick ones. In the present experimental case $\rho$ equals 20 , hence almost all the vibration visible is the slow one with the negligibly small damping coefficient. On the other band, by equation (47) we see that the amplitudes of the slow and quick vibrations of the light bob are numerically equal. Consequently the large damping coefficient, which is 20 times the small one, affects at least half of the amplitude visible.

Logarithmic decrements.-The lower trace on fig. 11 just dealt with, led to the theoretical introduction of the damping of the light bob as expressed by the constant $k$ in equation (4). It also became necessary to estimate the experimental value of $k$. To do this one pendulum with a light bob was allowed to oscillate alone, the other being meanwhile disconnected. The traces for the lighter bobs $\mathrm{P}$ were taken when their masses were respectively as used in the experiments, so as to be one-twentieth and one-fifth of those of the corresponding heavy ones. The results are given in fig. 12. From the upper trace with the very light bob consisting simply of a cardboard funnel, a few weights and sand (total mass about 50 gms.), we find that the logarithmic decrement is of the order $\lambda=0.017$.

Then by (57) we have

$$
k=\frac{m \lambda}{\pi}=(0.005) m \text {. }
$$

The lower trace with bob about 120 gms. shows considerably less damping and the decrement need not be evaluated. Masses 5:1-The masses of the bobs used in these experiments were of the order $600 \mathrm{gms}$. and $120 \mathrm{gms}$. respectively.

Figs. 13-19 in Plate II. show double traces obtained with this arrangement. In figs. 13-16 we see very plainly the beat effects on the lower trace which is left by the lighter bob. The traces of the heavier bob also show distinct but much slighter fluctuations of amplitude. In this respect they are seen to present an intermediate state between the cases of equal masses and masses as $20: 1$. And this is just what we should naturally expect. Further, the beat cycles contain fewer and fewer vibrations as the coupling increases. 
This again is in accord with theory, for the frequencies of the superposed vibrations are then more unequal and therefore gain more quickly on each other.

Lengths $3: 4$.-Figs. 20-28 show double traces simultaneously obtained with the masses of the bobs equal, but the lengths of the suspensions as $3: 4$. The lower trace on each figure is that made by the shorter pendulum. In the case of fig. 20, the short pendulum was pulled aside, the long one hanging still in its slightly displaced position. In the cases of figs. 21-25, the long one was drawn aside while the short one hung at rest in its more or less displaced position. In figs. 26-28 the long pendulum was pulled aside while the short one was held in its zero position, as this favoured the exhibition of the compound harmonic trace which it was then sought to obtain.

The couplings in this set vary from about 5 per cent. to over 60 per cent. In the 5 and 10 per cent. couplings the response of the second pendulum is feeble and the beat cycles contain very few vibrations. These are the effects of the inequality of lengths. But as the coupling is further increased these effects of the inequality of the separate frequencies are seen to be overpowered. This is exactly in accord with the theory as exhibited in the graphs on fig. 2.

Fig. 26 shows an accidental collision of the lighter bot with the releasing apparatus. But the effect of the blow is seen to pass away after a few vibrations, as shown by comparison with fig. 27 , which is a repetition of the conditions first intended. Figs. 26 and 27 are seen to present almost the appearance of the compound harmonic motion of a tone and its octave, the latter being too sharp. Fig. 28 shows the coupling reduced to 60 per cent., and this gives the relation of frequencies almost exactly $2: 1$.

The pair of simultaneous traces in fig. 28 is almost identical in type with those in fig. 11 of Plate V. in the October paper, in which latter case the lengths were equal. It may well seem surprising that the effect of the present mistuning (in which the frequency ratio exceeds $8: 7$ ) should be so completely obliterated by this coupling. But experiment and theory agree that it should be so.

\section{Summary.}

1. This second paper describes further experiments with the double-cord pendulum, but with the masses unequal as $20: 1$ and as $5: 1$, or the lengths unequal as $3: 4$. These 
are somewhat analogous to coupled electrical circuits with different inductances or different periods.

2. The case of masses $20: 1$ is seen to be very nearly that of forced vibrations in which the light bob is driven by receiving energy from the heavy bob or driver, while the latter's loss, though equal in energy, entails only a very small decrease of amplitude. The case of masses as $5: 1$ is about midway in character between that of $20: 1$ and equal masses. Eighteen photographic reproductions of double traces are given for unequal masses.

3. It was noticeable on one of the traces that the light bob showed diminntion of amplitude as the trace proceeded. This led to taking resistance into account in the equation of motion. It was also necessary to determine experimentally the actual damping of the light bob when vibrating separately. The theory thus developed and numerically applied fitted the observed facts.

4. In the case of unequal lengths but equal masses, a feebler response and a shorter beat cycle may naturally be expected than if mistuning were absent. Both these effects are quite striking with loose couplings. But with the tighter couplings the effect of mistuning is practically unnoticeable. The theory agrees with this experimental result. Nine sets of double traces are given for the nnequal periods.

5. It is hoped that these methods may be shortly applied to the illustration of important phenomena in other branches of Physics.

Nottingham,

Nov. 19,1917 .

IX. On the Diffraction of Light by Cylinders of Large Radius. By Nalinimohan Basu, M.Sc., Sir Rashbehari Ghosh Research Scholar in the University of Calcutta *.

[Plate III.]

\section{Introduction.}

1. C. F. BRUsh has recently published a paper containing some interesting observations on the diffraction of light by the edge of a cylindrical obstacle $\uparrow$. Brush worked with

* Communicated by Prof. C. V. Raman.

† "Some Diffraction Phenomena: Superposed Fringes," by C. F. Brush. Proceedings of the American Philosophical Society, 1913, pp. 276-282. See also 'Science Abstracts,' No. 1810 (1913). 
Phil. Mag. Ser. 6, Vol. 35, Pl. I.

$\bullet$

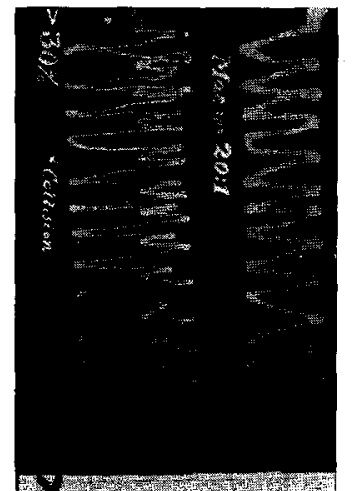

$\check{\circ}$

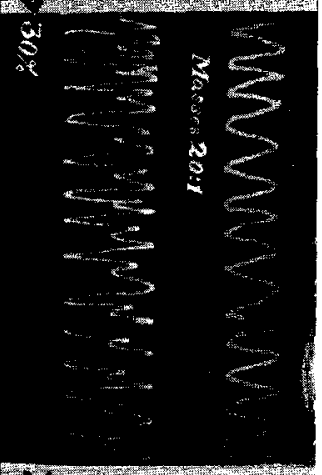

F
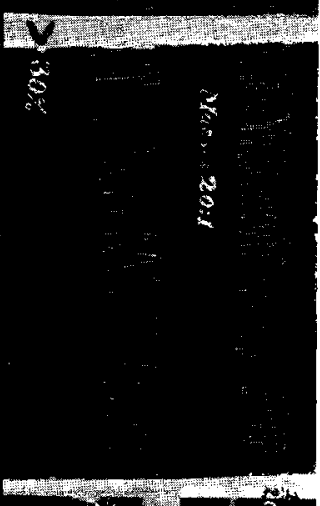

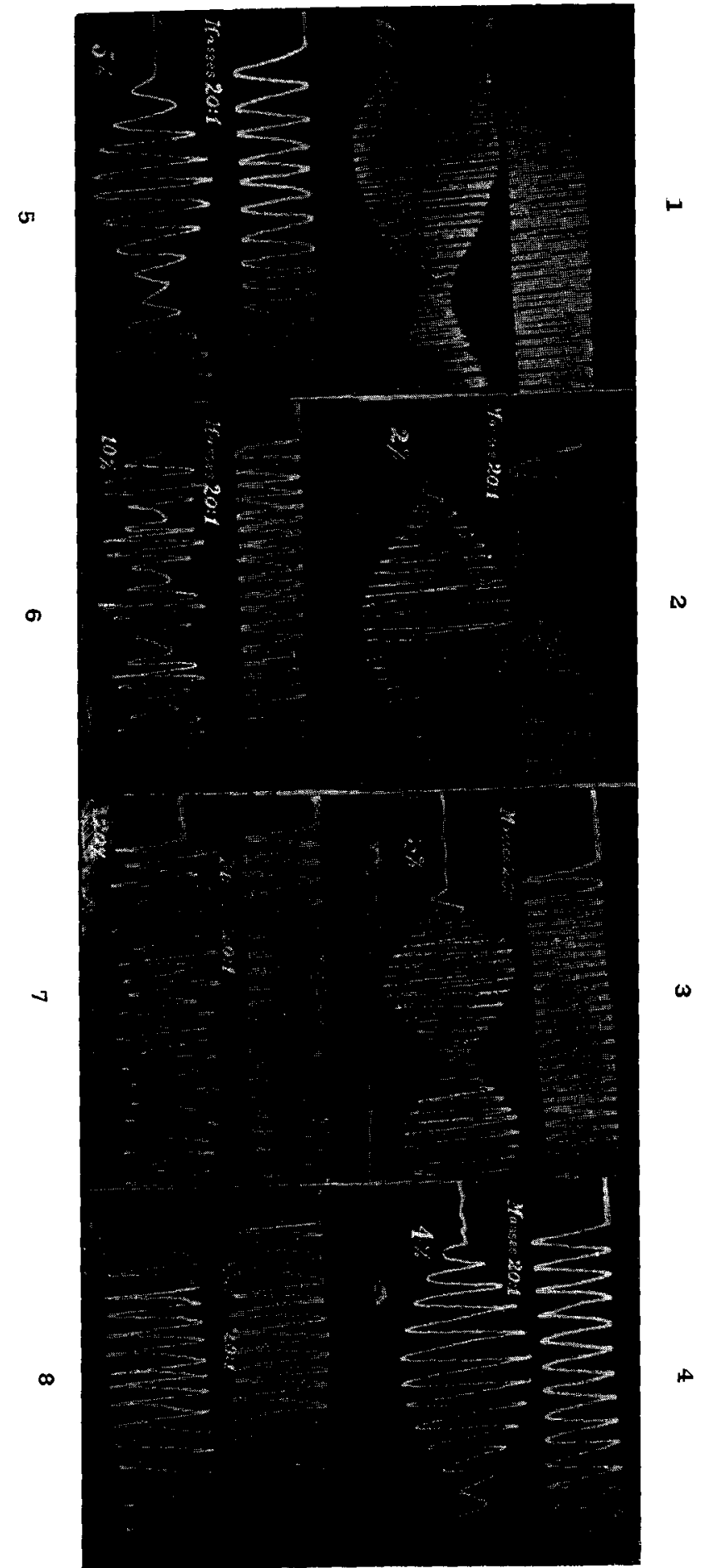


Phil. Mag. Ser. 6, Vol. 35, Pl. II.
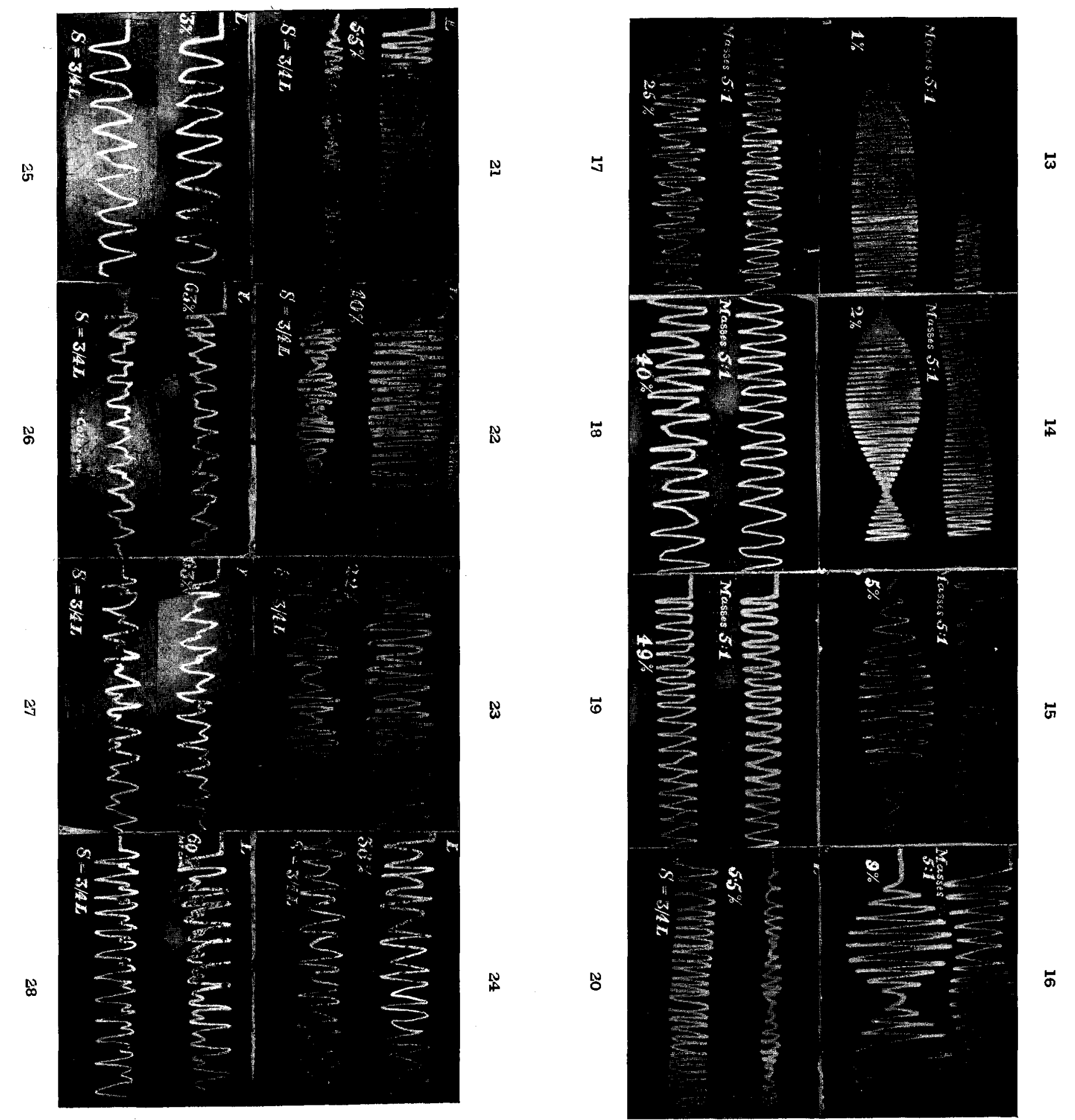